\title{
Evaluation of the relationship between subacromial space volume and rotator cuff injury by Magnetic Resonance Imaging with SPACE technique
}

\section{Safiye Sanem Dereli Bulut, Zakir Sakci}

Department of Radiology, Umraniye Research and Training Hospital, TC.Health Sciences University, Istanbul, Turkey

Received: 2021-03-01.

Accepted: 2021-04-28

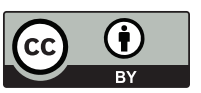

This work is licensed under a Creative Commons Attribution 4.0 International License

\section{J Clin Med Kaz 2021; 18(3):35-39}

Corresponding author:

Safiye Sanem Dereli Bulut.

E-mail: ssanembulut@gmail.com;

ORCID: 0000-0003-4593-6227

\begin{abstract}
Aim: To investigate the relationship between the degree of tearing in the rotator cuff tendons and the subacromial region volume using the proton-density weighted Sampling Perfection with Application optimized Contrasts using different flip angle Evolution technique and the contribution of this technique to shoulder magnetic resonance imaging.

Material and methods: A total of 125 patients who underwent shoulder magnetic resonance imaging for shoulder pain between January 2016 and December 2016 were included in this single-center, retrospective study. Rotator cuff tendon injury grading was performed using coronal plane 2D short tau inversion recovery T2-weighted sequences (grades 1-4). Subacromial region volume was calculated from PD-weighted 3D SPACE sequence images in sagittal oblique plane. The relationship between rotator cuff tears and subacromial region volumes was evaluated using Pearson's correlation analysis. The measurements were made at different times by two independent radiologists.

Results: There was a strong negative correlation between the grade of RC tendon tear and subacromial volume $(p<0.05 ; \mathrm{r}:-0.83)$.

Discussion and conclusion: Shoulder magnetic resonance imaging with high-contrast resolution can be obtained by the Sampling Perfection with Application optimized Contrasts using different flip angle Evolution technique. The degree of rotator cuff tendon tear, which is a cause of shoulder pain, is associated with the subacromial region volume. Using the Sampling Perfection with Application optimized Contrasts using different flip angle Evolution technique can also easily reveal the cause of the damage.
\end{abstract}

Key words: rotator cuff, shoulder, magnetic resonance imaging, SPACE technique

\section{Introduction}

Rotator cuff (RC) tendon pathologies occur widely [1-3], and there are many reasons for their etiology. The development and progression of RC injury remain uncertain, particularly when associated with external shoulder compression [1-3]. Traditionally, certain acromial morphologies have been thought to physically reduce the subacromial area and contribute to RC injury [1-3]; however, the relationship between subacromial space volume (SAV) and RC tearing has never been experimentally confirmed [1-3].
In 1972, Neer coined the term subacromial impingement and proposed a pathomechanical process in which mechanical compression of the soft tissues in the subacromial space occurred due to a narrowing of the subacromial space [4]. He asserted that the soft tissues most commonly involved was the bursal side of the supraspinatus and long head of biceps tendons which compress against the anterior and lateral edge of the acromion and coracoacromial ligament [4]. Neer proposed that any reduction of the subacromial space would lead to impingemnt syndrome [4]. 
The factors contribute to the shoulder impingement syndrome can be divided into extrinsic factors and intrinsic factors. Extrinsic factors that compress the structures with in the subacromial space (extra-tendinous), and intrinsic factors are those associated with degeneration of the rotator cuff tendons (intratendinous) [5].

In the literature, it is mentioned that the volume reduction of the subacromial region causes tendon degeneration by causing repetitive shear and compression forces [4]. It is also argued that histological changes in tendons, mechanical properties, morphology and vascularity of the tendon play a role in $\mathrm{RC}$ tendinopathy [6].

In this retrospective study, we measured the subacromial region volume of patients with $\mathrm{RC}$ tears by magnetic resonance imaging (MRI) and investigated whether it affects the degree of $\mathrm{RC}$ tearing.

\section{Material and methods Patients}

A total of 125 patients who had MRI performed in the radiology department due to shoulder pain between January 2016 and December 2016 were included in the study.

Ethics committee approval was obtained for this singlecenter retrospective study.

Patients with a history of shoulder trauma or surgery, inflammatory pathology, or infection were excluded from the study. Pediatric patients and patients with any contraindicated conditions (stenting, pregnant patients, claustrophobic patients, etc.) were also excluded from the study.

\section{MRI technique}

MRI of the shoulder was performed by a 1.5 Tesla device (Avanto; Siemens) while the patients were lying in the supine position with the shoulder joint in slight external rotation. The shoulder MRI sequence parameters are summarized in Table 1.

All were scanned using our routine 2D sequence protocol and the 3D Sampling Perfection with Application optimized Contrasts using different flip angle Evolution (SPACE) sequence.

\begin{tabular}{|c|c|c|c|c|c|}
\hline Table 1 & \multicolumn{5}{|c|}{$\begin{array}{l}\text { Shoulder magnetic resonance imaging } \\
\text { sequence parameters }\end{array}$} \\
\hline Sequence & 2D FS-PD & 2D FSTIR & T2 TSE & 2D T1-TSE & $\begin{array}{l}\text { 3D PD } \\
\text { SPACE }\end{array}$ \\
\hline Plane & Axial & $\begin{array}{l}\text { Coronal } \\
\text { oblique }\end{array}$ & $\begin{array}{l}\text { Sagittal } \\
\text { oblique }\end{array}$ & $\begin{array}{l}\text { Coronal } \\
\text { oblique }\end{array}$ & $\begin{array}{l}\text { Coronal } \\
\text { oblique }\end{array}$ \\
\hline TR (ms) & $\begin{array}{l}2000- \\
4000\end{array}$ & $>1500$ & $>2000$ & $400-800$ & 1100 \\
\hline TE (ms) & $30-50$ & $20-40$ & 90-110 & minimum & $30-50$ \\
\hline TI (ms) & - & 150 & - & - & - \\
\hline ETL & 8 & 8 & 8 & - & - \\
\hline BW & 16 & 16 & 16 & 16 & \\
\hline $\begin{array}{l}\text { Slice } \\
\text { thickness } \\
(\mathrm{mm})\end{array}$ & 4 & 3.5 & 3.5 & 3 & 0.7 \\
\hline $\begin{array}{l}\text { Interslice } \\
\text { gap (mm) }\end{array}$ & 1.2 & 1 & 1 & 0.5 & 0 \\
\hline Matrix & $256 \times 256$ & $256 \times 166$ & $256 \times 256$ & $256 \times 256$ & $256 \times 256$ \\
\hline FOV (mm) & 160 & 160 & 160 & 160 & 160 \\
\hline $\begin{array}{l}\text { Time } \\
\text { (min) } \\
\text { average }\end{array}$ & $1: 40$ & $1: 35$ & $1: 14$ & $1: 08$ & $7: 20$ \\
\hline
\end{tabular}

TSE: turbo spin echo image, SPACE: Sampling Perfection with Application optimized Contrasts using different flip angle Evolution, FS-PD: fat-suppressed proton densityweighted image, TE: echo time, TR: repetition time, FA: flip angle, FOV: field of view, ETL: echo train length, BW: bandwidth, ms: millisecond, mm: millimeter, min: minute

\section{Image analysis}

SAV measurements of the patients were made automatically in picture archiving and communication systems.

The initial diagnostic reading of the images was based on our routine 2D proton-density (PD) fat-suppressed (fs) protocol (Table 1). RC tendon tears were graded by the $2 \mathrm{D}$ short tau inversion recovery (STIR) technique, with fs T2-weighted Fast Spin Echo (FSE) sequences. In the visual evaluation, a tendon showing a T2 signal similar to the water signal was considered to be ruptured. A smaller T2 signal increase compared to the water signal was evaluated in favor of tendinosis and/or tendinopathy. This group of patients was excluded from the study.

The tendon tears were divided into three groups according to their location: tears extending to the joint surface of the tendon, tears extending to the bursal surface of the tendon, and tears within the tendon.

When classified according to the degree of tendon tear, the tendon thickness in the craniocaudal direction was graded as follows: If the tear was less than one-third of the tendon, it was accepted as a minimal partial tear (grade 1). If the tear was more than two-thirds of the tendon, an advanced partial tear was accepted (grade 3). Classifications between these two grades were accepted as a moderate partial tear (grade 2). If there was a loss of integrity in the tendon, it was accepted as a full-thickness tear (grade 4) (Figure 1a, b). Scoring increased with the degree of tendon tear.

Figure 1a. - 46-year-old female patient. The shoulder MRI performed for shoulder pain shows a moderate partial tear in the supraspinatus tendon on the bursal surface (thin blue arrow). It was observed in more than half the thickness of the torn tendon (small blue star) (grade 3).

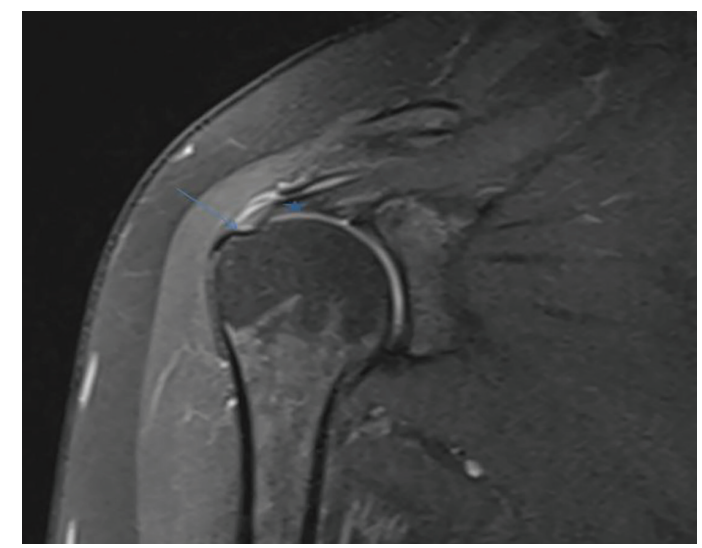

Figure 1b. - 48-year-old male patient. The supraspinatus tendon (small blue star) is not observed in the subacromial area in the shoulder MRI taken for shoulder pain. The supraspinatus (SS) tendon is seen as total ruptured. Proximal retraction of the ruptured fibers is observed (thin blue arrow).

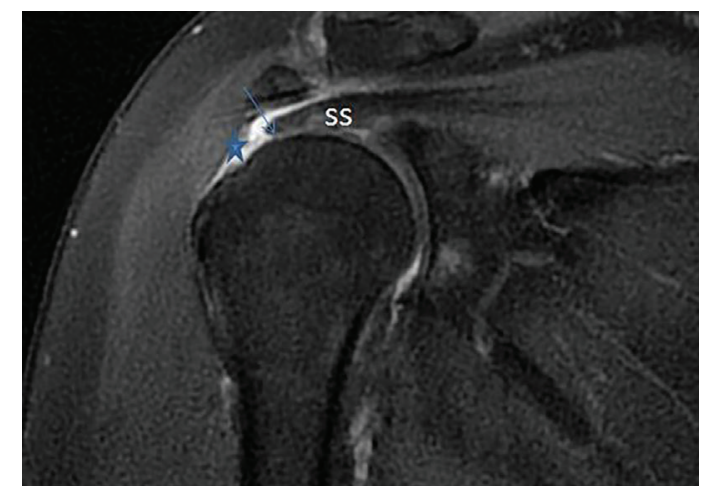




\section{Subacromial region volume calculation}

SAV measurements were made from the sagittal oblique plane PD-weighted 3D-SPACE sequence images. The area between the lower surface of the acromion and the chondral surface of the humeral head was calculated in serial sagittal oblique images from the acromial end to the acromioclavicular joint. The region of interest (ROI) areas marked on each section were automatically collected and multiplied by the number and thickness of the section, which automatically calculated the volume (cm3) of the subacromial region (Figure 2).

Two fully trained radiologists with 10 and 8 years' experience, respectively, in musculoskeletal imaging independently assessed all the exams. Diagnosis, gender, age, and clinical history were blinded for the reviewers.

Figure 2 - 38-year-old male patient. The volume measurement of the subacromial region is observed in the proton-density (PD) image made with the SPACE technique. A manually drawn ROI drawing with reference to the acromion lower cortex and the corresponding humeral joint face is shown. This process was repeated in every section where the acromion was visible. The drawn ROIs gave the result as an area; therefore, the result obtained was multiplied by the number and thickness of the sections, and the result was calculated as $\mathrm{cm} 3$.

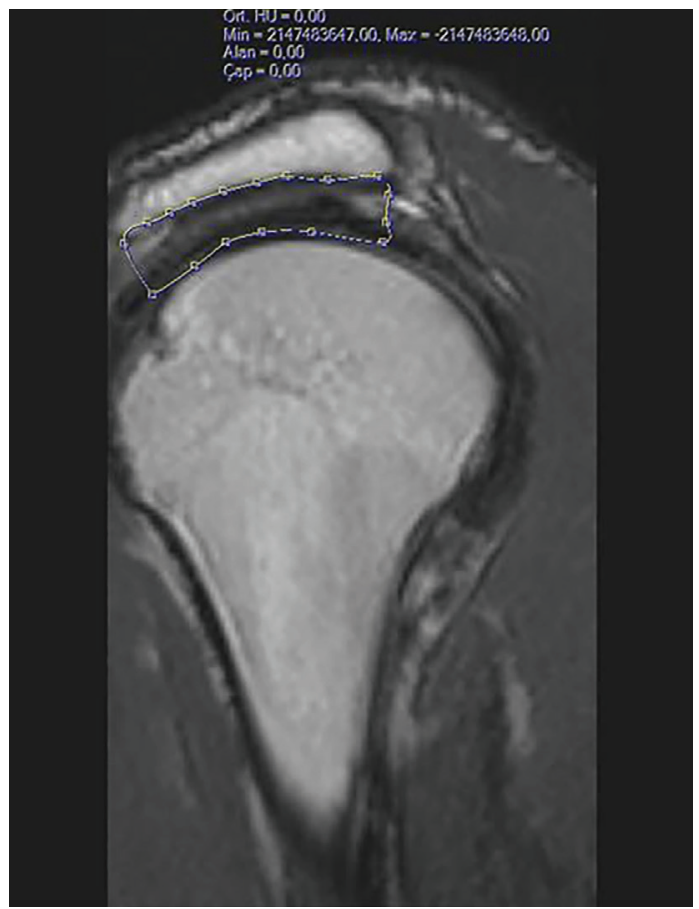

\section{Statistical analysis}

The distribution of outcome categories was assessed using the Shapiro-Wilk test. Data are presented as means \pm standard deviation based on the normality of the data. The categorical variables are reported as counts and percentages.

The relationship and degree of relationship between SAVs and $\mathrm{RC}$ tendon damage were tested by Pearson's correlation analysis. The correlation values $(\mathrm{r})$ were very poor $>0.25$; poor $0.25-0.50$; medium $0.50-0.70$; high $0.70-0.90$; or very high $0.90-1.00$.

All statistical analyses were performed using Statistical Package for the Social Sciences (SPSS) software (Version 22.0; SPSS Inc., Chicago, IL, USA).

A p-value $<0.05$ was considered statistically significant.

\section{Results}

The average age of the 125 patients included in the study was 52 years (48 years for women and 57 years for men).

The distribution percentages of the tendon tear classifications and the average SAVs in our patient population are summarized in Table 2.

There was statistically high agreement between the two observers in terms of RC tear classification ( $\mathrm{r}$ : 0.82).

There was a strong negative correlation between the grade of RC tendon damage and SAV ( $<<0.05 ; \mathrm{r}$ : -0.83) (Table 2).

The mean volumes for the subacromial region for mild partial tears were $3.48 \mathrm{~cm} 3$ for males and $3.16 \mathrm{~cm} 3$ for females and $2.52 \mathrm{~cm} 3$ and $2.47 \mathrm{~cm} 3$ for moderate partial RC tears, respectively. The mean volumes for full-thickness RC tears were $2.18 \mathrm{~cm} 3$ in men and $2.05 \mathrm{~cm} 3$ in women. The SAV measurements of the two observers were very consistent (r: 0.96).

\section{Discussion}

The RC consists of four muscles that come out of the scapula and attach to the humerus head. These muscles join as they pass the glenohumeral joint, adding strength and dynamic stability to the joint. The RC is important for shoulder motion $[4,5]$. The initiation of shoulder abduction relies on the function and integrity of the supraspinatus muscle and its tendon and other RC tendons [4-7].

During arm lift, the anterior acromion and the upper border of the subacromial space should move upward so that the head of the humerus is raised. If this does not happen, the rotator cuff tendon is compressed on the bursal side by the anterior acromion [4-7].

$\mathrm{RC}$ tendon tears occur in more than half of the population over the age of 60 . Shoulder pain is one of the most common musculoskeletal complaints, and shoulder impingement syndrome is the most commonly diagnosed shoulder disease [711]. The etiology of RC tears is multifactorial [7-11].

Morphology of the acromion has been considered to contribute to narrowing of the subacromial space, hence reducing the out let for the rotator cuff tendons. Bigliani and Levine evaluated acromion types and their frequencies in their study on 140 cadavers. They stated that Type 3 hooked acromion is the type most associated with RC tendon ruptures [7].

Narrowing in the subacromial area can be determined by direct or indirect measurement methods [12]. Indirect methods include acromion anterior slope, lateral acromial angle (LAA), acromion index (AI), acromio-glenoid angle (AGA), and critical shoulder angle. High slope angle, low LAA values, and increased AI are also associated with RC tears [12-14]. Tetreault et al. noted that low AGA values indicated a narrowing of the supraspinatus output [12-14]. According to theory, these measurement parameters show a decreased subacromial zone volume, and compression of the tendon in the subacromial region is an important finding [12-14].

Superficial migration of the humeral head in the chronic tears of RC tendons reduces the subacromial distance and thus the volume of the subacromial space $[13,14]$. Significant subacromial volume reduction in the early stage of RC tears indicates that humeral head migration has begun with the development of the RC tear $[13,14]$.

In the study of Lee et al., 40 fresh cadavers with and without RC tears were examined. According to the results of the study, no significant difference was found between the two cadaver groups in terms of acromion shape. As a result, it was 


\begin{tabular}{|l|l|l|l|l|l|l|}
\hline RC tear degrees & \multicolumn{2}{|l|}{$\begin{array}{l}\text { Number (n) and percentage (\%) of } \\
\text { patients }\end{array}$} & \multicolumn{2}{l|}{ Subacromial region volume (cm3) } & \multicolumn{3}{l|}{ Correlations between variant } \\
& n & $\%$ & Male & Female & $\begin{array}{l}\text { Significant } \\
(2 \text {-tailed) }\end{array}$ & Pearson correlation \\
\hline Grade 1 & 26 & 21 & $3.48 \pm 1.9$ & $3.16 \pm 0.6$ & 0,002 & $-0,93$ \\
\hline Grade 2 & 35 & 28 & $2.52 \pm 1.3$ & $2.47 \pm 1.4$ & 0,034 & $-0,83$ \\
\hline Grade 3 & 39 & 31 & $2.24 \pm 0.6$ & $2.21 \pm 1.2$ & 0,04 & $-0,87$ \\
\hline Grade 4 & 25 & 20 & $2.18 \pm 0.5$ & $2.05 \pm 0.8$ & 0,00 & $-0,96$ \\
\hline
\end{tabular}

concluded that "factors other than the acromion shape may play a role in the pathogenesis of rotator cuff tears" [15].

Other studies on subacromial decompression surgery, where the subacromial burs were excised, showed that the results were not different, whether performed with or without acromioplasty $[13,14]$. As a result, the idea that acromion morphology has nothing to do with subacromial impingement syndrome and this view is supported by many authors has been supported. Based on the available evidence from studies in the literature, the hypothesis that a reduction in subacromial area is a cause in impingement has not been firmly established $[16,17]$.

Independent of all these results, detailed imaging of the $\mathrm{RC}$ and subacromial region is extremely important in terms of elucidating the etiology $[18,19]$.

Computed tomography (CT) examination allows for high temporal resolution $[3,14]$; however, when evaluated in terms of contrast resolution, MRI is superior. In addition, contrast resolution is very important in a joint with complex anatomy, such as the shoulder joint. MRI is the gold standard imaging examination for the presence of intra-articular fluid, evaluation of bicipital labral pathologies, and detailed evaluation of muscle tissue and ligament pathologies $[18,19]$. The absence of radiation exposure and any invasive procedure in the evaluation of most intra-articular pathologies are other advantages $[18,19]$.

Surgical treatment may be considered in symptomatic $\mathrm{RC}$ tears and subacromial impingement that do not respond to conservative therapy $[2,3]$. Therefore, SAV measurement maybe a guide at this point.

The images we obtained with the SPACE sequence had high-contrast resolution, and we easily obtained reformatted images in $3 \mathrm{D}$ planes with the thin sections we obtained. We were also able to create oblique plane formats of these. In this way, we were able to follow each tendon throughout its entire course $[18,19]$.

Besides visual information about the shoulder junction muscle and bone tissue, measurements can also be made using shoulder MRI with appropriate position and technique. High- resolution images with very thin slice thicknesses can be obtained with CT. Images can be obtained in the axial plane, with a slice thickness of $1-3 \mathrm{~mm}$ that partially overlapped each other, and reconstructed to obtain thin sections $[18,19]$. Thus, 3D images can be obtained. Using the SPACE MR technique, a section thickness of $0.7 \mathrm{~mm}$ was obtained. The advantage of this examination is that the patient does not experience radiation exposure $[18,19]$.

Our study has some limitations. First, we did not classify patients according to age. In the advanced age group, fat atrophy in the $\mathrm{RC}$ and deltoid muscle is a finding that may contribute to impingement findings [1-3]. Consequently, the subacromial volume of older patients may be further decreased compared to younger patients. This issue may be explored in a future study. Second, this study had a small population and lacked clinical diversity. The third limitation is that patients with a diagnosis of subacromial impingement and subacromial volume measurement did not have arthroscopic findings. Clinical follow-up data for these patients were not available.

\section{Conclusion}

In conclusion, we were able to show the shoulder and subacromial region in MRIs with very thin slice thicknesses in three planes. We found that the degree of RC tendon damage, one of the causes of shoulder pain, correlated with the volume of the subacromial area. Images with high-contrast resolution can be obtained with shoulder MRI obtained using the SPACE technique. In this way, the etiology of shoulder pain can be easily illuminated.

Disclosures: There is no conflict of interest for all authors.

Acknowledgements: None.

Funding: None.

\section{References}

1. Kanatli U, Ayanog, T, Aktas E, Özer, M, Çetinkaya, M. Grade of coracoacromial ligament degeneration as a predictive factor for impingement syndrome and type of partial rotator cuff tear. J Shoulder Elbow Surg. 2016; 25(11):1824-1828. https://doi.org/10.1016/j. jse.2016.02.026

2. Daghir AA, Sookur PA, Shah S, Watson M. Dynamic ultrasound of the subacromial-subdeltoid bursa in patients with shoulder impingement: a comparison with normal volunteers. Skeletal Radiol. 2012; 41(9):1047-53. https://doi.org/10.1007/s00256-011-1295-Z

3. Moosikasuwan JB, Miller TT, Burke BJ. Rotator Cuff Tears: Clinical, Radiographic, and US Findings. RadioGraphics. 2005; 25:15911607. https://doi.org/10.1148/rg.256045203

4. Neer, C.S.I., 1983. Impingement Lesions. Clinical Orthopaedics \& Related Research. 1983; 70-77. https://doi.org/10.1097/00003086198303000-00010

5. Seitz, A.L., McClure, P.W., Finucane, S., Boardman III, N.D., Michener, L.A., 2011. Mechanisms of rotator cuff tendinopathy: Intrinsic, extrinsic, or both? Clinical Biomechanics. 26:1-12. https://doi.org/10.1016/j.clinbiomech.2010.08.001

6. Girish G, Lobo LG, Jacobson JA, Morag Y, Miller B, Jamadar DA. Ultrasound of the Shoulder: Asymptomatic Findings in Men. AJR. 2011; 197:W713-W719. https://doi.org/10.2214/AJR.11.6971 
7. Bigliani, L.U., Codd, T.P., Connor, P.M., Levine, W.N., Littlefield, M.A., Hershon, S.J., 1997. Shoulder Motion and Laxity in the Professional Baseball Player. Am J Sports Med. 25:609-613 https://doi.org/10.1177/036354659702500504

8. Craik JD, Mallina R, Ramasamy V, Little, NJ. Human evolution and tears of the rotator cuff. Int Orthop. 2014; 38(3):547-552. https:// doi.org/10.1007/s00264-013-2204-y

9. Durmaz MS, Karahan Y. Omuz Ultrasonografi İncelemesi Manyetik Rezonans Görüntüleme' nin Yerini Alabilir Mi? [in Turkish]. Dicle Med J. 2020; 47:929-939. https://doi.org/10.5798/dicletip.850507

10. Teefey SA, Middleton WD, Payne WT, Yamaguchi K. Detection and Measurement of Rotator Cuff Tears with Sonography: Analysis of Diagnostic Errors. AJR. 2005; 184:1768-1773. https://doi.org/10.2214/ajr.184.6.01841768

11. M. J. Tuite MJ, Small KM. Imaging Evaluation of Nonacute Shoulder Pain. AJR. 2017; 209:525-533. https://doi.org/10.2214/ AJR.17.18085

12. Pepe M, Kocadal O, Gunes Z, Calisal E. Subacromial space volume in patients with rotator cuff tear: The effect of surgical repair. Acta Orthop. Traumatol. Turc. 2018; 52(6):419-422. https://doi.org/10.1016/j.aott.2018.08.003

13. Nyffeler RW, Meyer DC. Acromion and glenoid shape: Why are they important predictive factors for the future of our shoulders? EFORT Open Rev. 2017; 2(5):141-150. https://doi.org/10.1302/2058-5241.2.160076

14. Titreault P, Krueger A, Zurakowski D, Gerber C. Glenoid version and rotator cuff tears. J Orthop Res. 2004; 22(1):202-207. https://doi. org/10.1016/S0736-0266(03)00116-5

15. Lee SB, Itoi, E., O'Driscoll, S.W., An, K.-N., 2001. Contact geometry at the undersurface of the acromion with and without a rotator cuff tear. Arthroscopy: The Journal of Arthroscopic \&Related Surgery. 17:365-372. https://doi.org/10.1053/jars.2001.19974

16. Budoff, J.E., Rodin, D., Ochiai, D., Nirschl, R.P., 2005. Arthroscopic Rotator Cuff Debridement Without Decompression for the Treatment of Tendinosis. Arthroscopy: The Journal of Arthroscopic \& Related Surgery. 21:1081-1089. https://doi.org/10.1016/j.arthro.2005.05.019

17. Henkus, H.E., Witte, P.B. de, Nelissen, R.G.H.H., Brand, R., Arkel, E.R.A. van, 2009. Bursectomy compared with acromioplasty in the management of subacromial impingement syndrome a prospectıve randomised study. J Bone Joint Surg Br. 91-B:504-510. https://doi. org/10.1302/0301-620X.91B4.21442

18. Karsten J, et al. Comparison of 3D turbo spin-echo SPACE sequences with conventional 2D MRI sequences to assess the shoulder joint. Eur. J. Radiol. 2014; 83(1):1843-1849. https://doi.org/10.1016/j.ejrad.2014.06.011

19. Henninger B, Raithel E, Kranewitter C, Steurer M, Jaschke W, Kremser C. Evaluation of an accelerated 3D SPACE sequence with compressed sensing and free-stop scan mode for imaging of the knee. Eur. J. Radiol. 2018; 102:74-82. https://doi.org/10.1016/j. ejrad.2018.03.001 